\title{
Análise do conhecimento de internos sobre interpretação e conduta da dislipidemia em uma faculdade de Teresina-PI
}

Analysis of the knowledge of interns about the interpretation and conduct of dyslipidemia in a faculty of Teresina-PI

Análisis del conocimiento de internos sobre la interpretación y conducta de dislipidemia en una facultad de Teresina-PI

\author{
Miguel Tourinho Azevedo \\ ORCID: https://orcid.org/0000-0002-4329-8594 \\ Centro Universitário Uninovafapi, Brasil \\ E-mail: Azevedo.tmiguel@gmail.com \\ Thauan Castelo Branco Ferreira Carvalho \\ ORCID: https://orcid.org/0000-0001-9758-8899 \\ Centro Universitário Uninovafapi, Brasil \\ E-mail: thauancarvalho11@gmail.com \\ Williams Cardec da Silva \\ ORCID: https://orcid.org/0000-0002-7167-4876 \\ Centro Universitário Uninovafapi, Brasil \\ E-mail: willcardec@hotmail.com
}

\begin{abstract}
Resumo
Os exames bioquímicos de perfil lipídico são a forma de investigação de dislipidemia, um dos principais fatores de risco para as doenças cardiovasculares. Profissionais médicos devem saber quando corretamente solicitar esses exames, interpretá-los e tomar condutas para redução dos fatores de risco modificáveis e da dislipidemia em si para, então, oferecer uma assistência adequada ao paciente. Essa pesquisa objetivou avaliar e comparar o conhecimento dos acadêmicos de medicina sobre o assunto. Trata-se de um estudo descritivo, observacional e transversal, realizado com 116 alunos do curso de medicina de uma faculdade em Teresina-PI que estavam regularmente matriculados no internato (do $9^{\circ}$ ao $12^{\circ}$ período), coletados através de um questionário online com um total de 13 questões. Os resultados indicaram uma média de acertos crescente quanto maior o período em curso, além de maior domínio quando o aluno já havia cursado o ciclo de Atenção Básica. Contudo, os resultados gerais refletiram ainda um conhecimento reduzido sobre a dislipidemia e suas condutas.
\end{abstract}

Palavras-chave: Dislipidemias; Medicina; Conhecimento; Estudantes.

\begin{abstract}
The lipid biochemical tests are the tool to investigate dyslipidemia, one of the main risk factors for cardiovascular diseases. Medical professionals must know when to correctly order these tests, interpret them and take steps to reduce modifiable risk factors and dyslipidemia itself, in order to provide adequate care. This research aimed to evaluate and compare the knowledge of medical students on the subject. This is a descriptive, observational and cross-sectional study, carried out with 116 medical students of a college in Teresina-PI who were regularly enrolled in the internship (from 9th to 12th period), collected through an online questionnaire with 13 questions. The results indicated an increasing average of correct answers the longer the period in progress, in addition to greater mastery when the student had already attended the Primary Care cycle. However, the overall results still reflected little knowledge about dyslipidemia and its behavior.
\end{abstract}

Keywords: Dyslipidemias; Medicine; Knowledge; Students.

\section{Resumen}

Las pruebas bioquímicas de perfil lipídico son la forma de investigar la dislipidemia, uno de los principales factores de riesgo de las enfermedades cardiovasculares. Los profesionales médicos deben saber cuándo solicitar correctamente estas pruebas, interpretarlas y tomar las medidas necesarias para reducir los factores de riesgo modificables y la propia dislipidemia, para luego brindar una atención adecuada al paciente. Esta investigación tuvo como objetivo evaluar y comparar los conocimientos de los estudiantes de medicina sobre el tema. Se trata de un estudio descriptivo, observacional y transversal, realizado con 116 estudiantes de medicina de una facultad de Teresina-PI que estaban matriculados habitualmente en la pasantía (del 9 al 12 período), recogidos a través de un cuestionario online con un total de 13 preguntas. Los resultados indicaron un promedio creciente de aciertos cuanto mayor era el período en curso, 
además de un mayor dominio cuando el alumno ya había cursado el ciclo de Atención Primaria. Sin embargo, los resultados generales aún reflejan poco conocimiento sobre la dislipidemia y su comportamiento.

Palabras clave: Dislipidemias; Medicina; Conocimiento; Estudiantes.

\section{Introdução}

Os lipídeos exercem funções fundamentais na absorção de nutrientes lipofílicos, como as vitaminas, além de atuarem no armazenamento e na produção de energia (Gusso, Lopes \& Dias, 2019)

Um perfil lipídico completo é composto por: colesterol total, triglicerídeos, colesterol de lipoproteína de alta densidade (HDL-C) e colesterol de lipoproteína de baixa densidade (LDL-C), e tanto do ponto de vista fisiológico quanto clínico, são também os mais relevantes. (Rosenson, 2020; Faludi, Izar \& Saraiva, 2017)

As dislipidemias são definidas como distúrbios no metabolismo das lipoproteínas, como o aumento do colesterol total, da lipoproteína de baixa densidade (LDL) e dos triglicerídeos, e diminuição da lipoproteína de alta densidade (HDL), sendo desenvolvidas de acordo com a exposição a fatores genéticos e/ou ambientais. (Souza, 2019)

Segundo a última atualização da Diretriz Brasileira de Dislipidemia e Aterosclerose da Sociedade Brasileira de Cardiologia, em sua última atualização sobre o perfil lipídico, de 2017, constou que os valores de referência para maiores de 20 anos são: Colesterol total < $190 \mathrm{mg} / \mathrm{dL}, \mathrm{HDL}>40 \mathrm{mg} / \mathrm{dL}$, triglicerídeos $<150 \mathrm{mg} / \mathrm{dL}$. O valor dos triglicerídeos são os únicos que se alteram com o estado de jejum e de não jejum. As dislipidemias podem, assim, ser classificadas laboratorialmente de acordo com a fração lipídica alterada em:

- Hipercolesterolemia isolada: aumento isolado do LDL (LDL-c $\geq 160 \mathrm{mg} / \mathrm{dL}$ ).

- Hipertrigliceridemia isolada: aumento isolado das triglicérides ( $\mathrm{TG} \geq 150 \mathrm{mg} / \mathrm{dL}$ ou $\geq 175 \mathrm{mg} / \mathrm{dL}$, se a amostra for obtida sem jejum de 12 horas).

- Hiperlipidemia mista: aumento do LDL e dos TG. Se TG $\geq 400 \mathrm{mg} / \mathrm{dL}$, o cálculo do LDL-c pela fórmula de Friedewald é inadequado, devendo-se considerar a hiperlipidemia mista quando o não HDL-c $\geq 190 \mathrm{mg} / \mathrm{dL}$.

- HDL-c baixo: redução do HDL-c (homens $<40 \mathrm{mg} / \mathrm{dL}$ e mulheres $<50 \mathrm{mg} / \mathrm{dL}$ ) isolada ou em associação ao aumento de LDLc ou de TG.

Os triglicerídeos são formados a partir de três ácidos graxos livres ligados a uma molécula de glicerol e é uma das mais importantes formas de armazenamento energético. Eles podem ser provenientes de fontes exógenas (dieta) e de fontes endógenas (síntese hepática). Além disso, a hipertrigliceridemia está relacionada ao consumo excessivo de gorduras saturadas (em detrimento das gorduras mono e polinsaturadas), de carboidratos simples e de álcool. (Torres, 2017)

O HDL-C, ao contrário do LDL-C, tem propriedades antiaterogênicas que incluem o transporte reverso do colesterol, a manutenção da função endotelial e a proteção contra a trombose. (Zhao, 2020) Devido a isso, o ideal é que o valor do HDL esteja acima do limite de inferioridade.

A redução dos níveis de colesterol não HDL já é aceita como fator importante na diminuição das DCVs, como demonstraram diversos estudos e metanálises. Da mesma forma, o aumento do HDL é visto como fator de proteção. (Gusso et al., 2019).

A estratificação de risco de dislipidemia faz-se mister para o cálculo do risco cardiovascular. O manejo dos fatores de risco para DCVs, entre os quais o LDL-C elevado, é chamado de prevenção primária se esse processo for feito em alguém que não experimentou anteriormente um evento vascular aterosclerótico. (Pignone, 2020)

Além disso, a dislipidemia, em conjunto com o tabagismo, sobrepeso, dieta pouco saudável, sedentarismo, hipertensão e diabetes mellitus formam os principais fatores de risco para DCVs, sendo modificáveis e considerados para intervenção em todos os pacientes adultos. (Hennekens, 2020) 
No contexto das DCVs (Doenças Cardiovasculares), elas são a primeira causa de óbito no mundo e no Brasil, onde corresponderam a $27 \%$ das causas de morte no ano de 2015. (OMS, 2018). Com o envelhecimento populacional, a doença cardíaca isquêmica e a doença cerebrovascular têm ganhado importância crescente não apenas como causas de morte, mas também de morbidade e incapacidade funcional. (Vos, 2016; Adamson, Beswick \& Ebrahim, 2004)

A avaliação do RCV depende de informações obtidas na história clínica, no exame físico e através de exames complementares, sempre objetivando a identificação de coexistência de outros fatores de risco, presença de lesão de órgão-alvo e diagnóstico de doença cardiovascular ou doença renal já estabelecida. (Malachias, 2016)

Como repercussões clínicas, a dislipidemia é uma das complicações mais frequentes da obesidade. Trata-se de distúrbio metabólico caracterizado por concentrações anormais de lipídeos e/ou lipoproteínas no sangue, sendo determinada por fatores genéticos e ambientais. (Suen, Durval \& De Almeida, 2017)

As taxas excessivas são prejudiciais, visto que o aumento dos seus níveis séricos está associado a aterosclerose. (Gusso et al, 2019)

A aterosclerose é caracterizada pela lesão do endotélio, ocasionando o depósito de gordura, LDL-colesterol oxidado, células de espuma, cálcio e fibrina nos revestimentos internos da parede arterial, provocando estreitamento do canal sanguíneo que oxigenam o músculo cardíaco. A placa, aterosclerose, pode sofrer ruptura causando a formação de um coágulo sanguíneo que bloqueia a artéria ou outra parte do corpo por onde circular, por esse motivo, há possibilidade de ocorrer o infarto agudo do miocárdio (IAM), ou acidente vascular encefálico (AVE). (Santos, Dos Santos \& Dias, 2021)

É um processo patológico que causa doenças nas artérias coronárias, cerebrais, periféricas e na aorta. (Zhao, 2020)

Vale ressaltar que a dislipidemia pode ocorrer em adultos jovens (definidos como pessoas com idade entre 21 e 39 anos) e é um importante fator de risco para doenças cardiovasculares. O rastreamento pode identificar adultos jovens com dislipidemia assintomática que podem se beneficiar de terapias hipolipemiantes. (Chou, 2016)

Mudança de estilo de vida é recomendado para todos os pacientes com risco cardiovascular aumentado, e incluem exercícios regulares, parar de fumar, atingir um peso corporal alvo e uma dieta saudável. (Rosenson, 2020)

A prática de exercícios físicos é considerada como uma das principais intervenções não farmacológicas contra a dislipidemia e doenças vasculares, pois exerce influência na melhora do perfil lipídico, condutância vascular, complacência arterial, modulação autonômica com melhora do balanço simpatovagal, diminuição dos níveis de pressão arterial, redução dos níveis de gordura corporal, normalização da glicemia, entre outras respostas benéficas. (Soares, 2018)

O monitoramento periódico, como por meio de exames complementares, é fundamental, incluindo a divulgação das ações e metas alcançadas, de forma a apoiar o Governo Brasileiro e a sociedade civil no acompanhamento do Plano de combate as DCNTs e fortificar os compromissos entre governos e comunidade, a fim de reduzir sua morbimortalidade e melhorar a situação de saúde da população brasileira. (Malta, 2016)

Já em relação ao uso de medicações, as estatinas agem inibindo a atividade da enzima HMG-CoA redutase, não permitindo formação de mevalonato, o que acarreta a redução na síntese hepática do colesterol e, como consequência, aumento da síntese dos receptores de Lipoproteínas de Baixa Densidade (LDL, sigla do inglês Low Density Lipoprotein) nos hepatócitos, aumentando, assim, sua captação da circulação para repor o colesterol intracelular. (Bonfim, 2015)

A terapia com estatinas reduzirá o risco relativo de eventos cardiovasculares em 20 a $25 \%$ quando usada para prevenção primária; portanto, aqueles com o risco de linha de base mais alto provavelmente receberão a maior melhora absoluta no risco com o tratamento. (Vijan, 2020)

Apesar do mecanismo de ação dos fibratos não estar completamente elucidado, eles apresentam dois mecanismos farmacológicos principais: redução da síntese hepática de triglicérides, pela inibição parcial da ação da lipólise periférica e do 
fluxo de ácidos graxos para o fígado e aumento no catabolismo das lipoproteínas ricas em triglicérides, devido à estimulação da lipase lipoprotéica. (Bertolami, 2018).

\section{Metodologia}

A pesquisa ocorreu no período de 01 maio de 2021 a 30 de julho de 2021, após a devida aprovação no Comitê de Ética e Pesquisa (CEP) do Centro Universitário UNINOVAFAPI. Foi feita de forma On-line, através da ferramenta Google Forms, na modalidade pesquisa de campo, sobre conhecimento, interpretação e conduta da dislipidemia. Para isto, foi elaborado um questionário social com as seguintes informações: gênero, idade e período letivo atual. Em seguida, um questionário específico contendo casos clínicos, sendo abordado conhecimento teórico e/ou prático quanto a interpretação de exames e conduta sobre dislipidemia. $\mathrm{O}$ formulário foi arquitetado da seguinte maneira:

- 13 questões objetivas, em que os autores e orientador do trabalho foram responsáveis pela montagem e pesquisa das questões.

- Após a coleta de dados da amostra, foram quantificadas e analisadas as respostas, sendo utilizados métodos visuais, como gráficos, para melhor interpretação desses dados.

A pesquisa foi incialmente projetada para uma amostra de 143 internos de medicina do Centro Universitário Uninovafapi, selecionados aleatoriamente entre o universo de 227 internos matriculados em 2021. Para determinar a amostra, foi usada a seguinte fórmula, conforme orientação de estatístico: $\mathrm{n}=\left(\mathrm{z}^{2} \cdot 0,25 . \mathrm{N}\right) /\left[\mathrm{E}^{2}(\mathrm{~N}-1)+\mathrm{z}^{2} \cdot 0,25\right]=\left(1,96^{2} \cdot 0,25 \cdot 227\right)$ / $\left(0,05^{2} \cdot 226+1,96^{2} \cdot 0,25\right)$, em que, z é o valor crítico, E é a margem de erro e $\mathrm{N}$ o tamanho da população. Considera-se o grau de confiança de $95 \%(z=1,96)$, margem de erro $\mathrm{E}=5 \%$ e $\mathrm{N}=227$.

Contudo, o número real de formulários coletados foi 118, dos quais 2 foram invalidados por erros de preenchimento. Assim, chegamos ao número final de 116 respostas válidas. Os dados foram tabulados em planilha eletrônica Microsoft Office Excel e analisados no próprio programa.

\section{Critérios de Inclusão}

- A amostra deve estar entre o $9^{\circ}$ período e $12^{\circ}$ período do curso de Medicina do Centro Universitário Uninovafapi;

\section{Critérios de Exclusão}

- Alunos que estudam no Centro Universitário Uninovafapi e que não se encontram no internato;

- Alunos de outras instituições de ensino;

- Internos que não estão devidamente matriculados.

\section{Resultados e Discussão}

Considerando o universo de 227 internos que cursam entre o $9^{\circ}$ e $12^{\circ}$ período do curso de medicina no Centro Universitário Uninovafapi (UNINOVAFAPI), no período de agosto a dezembro de 2021, o número da nossa amostra seria de 143. Porém, por fatores de antecipação de formatura e recusa das entrevistas, apenas 118 alunos foram entrevistados. Destes, 2 foram eliminados por problemas de preenchimento do questionário, totalizando uma amostra final de 116 participantes.

A amostra da pesquisa foi submetida a dois tipos de questionários: questionário social e questionário específico sobre a temática deste estudo, denominado aqui questionário específico. Sobre o questionário social, as perguntas destinadas aos internos 
eram referentes ao sexo, idade, período em curso no momento da entrevista e se já havia cursado o ciclo do internato programa saúde da família (PSF).

Referente ao sexo, 64 definiram ser do sexo feminino (55,2\%), 51 do sexo masculino (44\%) e 1 preferiu não informar (0,8\%). Quanto a idade, a amostra variava de 22 a 36 anos, apresentando o seguinte perfil: 22 anos, 13 (11,2\%); 23 anos, 17 (14,7\%); 24 anos, 17 (14,7\%); 25 anos, 32 (27,6\%); 26 anos, 15 (12,9\%); 27 anos, 6 (5,2\%); 28 anos, 5 (4,3\%); 29 anos, 5 $(4,3 \%)$; 30 anos, 4 (3,4\%); 31 anos, 1 (0,9\%); 36 anos, 1 (0,9\%). Quanto ao período, 37 internos (31,9\%) declararam que estavam no $9^{\circ}$ período; 21 internos $(18,1 \%)$ estavam no $10^{\circ}$ período; 49 internos $(42,2 \%)$ estavam no $11^{\circ}$ período; 9 internos $(7,8 \%)$ declararam que estavam no $12^{\circ}$ período. E em relação se já havia cursado o ciclo de PSF, 61 internos (52,6\%) ainda não cursaram; 24 internos (20,7\%) estavam atualmente no ciclo e 31 internos (26,7\%) já haviam finalizado.

Em relação ao questionário específico do tema deste estudo, compunha-se de 13 questões: a primeira seção com 2 perguntas de opinião e a segunda com 11 perguntas objetivas.

A seção de opinião questionava, em uma autoanálise, se o avaliado achava que sabia todos os temas pertinentes à identificação da dislipidemia em pacientes, em que 46 internos (39,7\%) responderam NÃO e 70 internos (60,3\%), SIM. Na segunda questão avaliava-se se a aluno saberia conduzir adequadamente um paciente com dislipidemia, em que apenas 14 internos $(12,1 \%)$ responderam que apresentavam domínio total sobre o assunto; 50 internos $(43,1 \%)$ responderam que tinham domínio parcial do assunto; 50 internos $(43,1 \%)$ relataram que precisavam revisar antes de conduzir um caso de dislipidemia e 2 internos $(1,7 \%)$ disseram que não saberiam conduzir adequadamente um caso de dislipidemia.

Após as questões de autoanálise, foram iniciadas as perguntas relacionadas a temática deste estudo. A seção objetiva, composta de 11 questões, inicia-se com 2 questões cobrando conceitos, classificação e parâmetros básicos pertinentes à dislipidemia. As duas primeiras questões dessa seção obtiveram, respectivamente, $70(60,3 \%)$ e $100(86,2 \%)$ de acertos.

A terceira questão traz conceitos de dislipidemia relacionados a um caso clínico em grupos especiais (mulheres em uso de ACO, idosos e diabéticos), correlacionando e interligando os fatores de risco entre patologias, muito comum na prática médica diária. Houve 90 respostas corretas $(77,6 \%)$.

A quarta e quinta questão trazem noções referentes aos parâmetros numéricos da dislipidemia, pedindo a identificação da classificação dentre os 4 tipos de dislipidemia. Houve 85,3\% de acertos - contabilizando 99 respostas corretas, na questão quatro, e $81,9 \%$ (95) de acertos na questão cinco.

A sexta questão é a primeira que avalia os conhecimentos acerca do tratamento farmacológico e não farmacológico de um paciente portador de dislipidemia. Envolve principalmente os conhecimentos referentes ao grupo de medicamentos mais disseminado no sistema único de saúde quanto no sistema particular: as estatinas. Os estudantes tiveram um rendimento de apenas $50,9 \%$ quando pesquisado pela alternativa correta -59 respostas corretas.

Já a sétima questão abordava sobre efeitos adversos do uso das estatinas e medidas para controle das repercussões, onde 48,3\% dos entrevistados (56 alunos) responderam corretamente que a dosagem de rotineira de CK não é recomendada quando há introdução de novo medicamento hipolipemiante ou elevação da posologia de estatinas.

Quanto a oitava questão, obtivemos a minoria de respostas corretas quando comparada as outras, mais uma questão abordando sobre condução terapêutica, apenas $22,4 \%$, com 26 respostas.

A $11^{\circ}, 12^{\circ}, 13^{\circ}$ questões abordaram um caso clínico amplo, muitos sinais clínicos para correlacionar sobre tratamento e fatores de risco, onde o rendimento dos internos foi de 48,3\% (56 alunos), 54,3\% (63 alunos) e 77,6\% (90 alunos), respectivamente.

Em uma análise mais aprofundada, podemos unir as informações coletadas e criar resultados a partir de suas intersecções. Ao dividir o grupo de alunos em Os que 'Não cursaram o ciclo de Atenção Básica" e os que "'Já finalizaram ou estão atualmente cursando o ciclo de Atenção Básica", considerando todos os períodos estudados. 
Somando os alunos que já cursaram ou que estão atualmente no ciclo de Atenção Básica, temos um universo de 55 alunos, contra 61 que ainda não cursaram. Comparando suas respostas, nota-se que há, percentualmente, um número maior de acertos quando analisadas todas as questões. Esse fato só não ocorreu nas questões de número 4, 5 e 10 (3 de um total de 11 questões). A questão 1 cursou com 67,27\% de acertos dos que cursaram comparados a 54,10\% dos que ainda não cursaram. De forma respectiva, a questão 2 teve $87,27 \%$ contra $85,25 \%$; a questão 3 teve $81,82 \%$ a $73,77 \%$. A questão 4 teve $83,64 \%$ contra 86,89\% (maior número de acertos do grupo que não cursou o ciclo de atenção básica). Na questão 5, 78,18\% contra 85,25\%; A questão 6 , com $58,18 \%$ contra $44,26 \%$; a questão 7 com $56,36 \%$ contra $40,98 \%$; a questão 8 com $25,45 \%$ contra $19,67 \%$; questão 9 com 58,18\% contra 39,34\%; a questão 10 com 50,91\% contra 57,38\%; e a questão 11 com 78,18\% contra 77,05\%. Nota-se, assim, que o ciclo no internato de atenção básica é importante e primordial para o desenvolvimento e fixação de conhecimentos relevantes para a suspeição, identificação, diagnóstico e correta tomada de conduta referente ao tema de dislipidemia. Os achados referentes as questões de números 4, 5 e 10 podem ser considerados de menor importância, visto que em todas as três questões, o percentual de acertos era alto em ambos os grupos, cerca de $80 \%$ de acerto.

Nota-se também uma melhora significativa entre os grupos de alunos nas questões 1, 3, 6, 7, 8 e 9. Houve um aumento percentual de $13,17 \%, 8,05 \% ; 13,92 \% ; 15,38 \% ; 5,78 \%$; e $18,84 \%$.

Analisando os grupos de alunos divididos em anos, pode-se classificá-los em $5^{\circ}$ ano (composto pelo $9^{\circ}$ e $10^{\circ}$ período) e $6^{\circ}$ ano (alunos dos $11^{\circ}$ e $12^{\circ}$ períodos). Os dois grupos possuem o mesmo número de 58 alunos em cada. Comparando os resultados, o $6^{\circ}$ ano apresentou rendimento superior quando comparado aos resultados do $5^{\circ}$ ano em todas as questões, exceto a $10^{\mathrm{a}}$ e $11^{\mathrm{a}}$. Na questão de número 1 , há $58,62 \%$ de acertos do $5^{\circ}$ ano quando comparado a $62,07 \%$ do $6^{\circ}$ ano. Respectivamente, há, na segunda questão, 82,76\% contra 89,66\%; questão 3 com 72,41\% contra 82,76\%; questão 4 com 82,76\% contra 87,93\%; questão 5 com 75,86\% contra 87,93\%; questão 6 com 39,66\% contra 62,07\% - uma diferença gritante. Questão 7 com 44,83\% pelo $5^{\circ}$ ano contra $51,72 \%$ do $6^{\circ}$ ano. De forma semelhante, a questão 8 com 10,34\% contra 34,48\%; questão 9 com 36,21\% contra $60,34 \%$; questão 10 com 56,90\% contra $51,72 \%$. E a questão 11 , com 79,31\% contra $75,86 \%$ de acertos pelo $6^{\circ}$ ano.

Aqui, também, pode-se concluir que a superioridade do número de respostas do $5^{\circ}$ ano referente as questões de número 10 e 11 é um achado menor, visto que são minoria de questões, estão ambos em uma porcentagem aceitável (próximo dos $80 \%$ de acerto) e estarem próximos por pequena margem de diferença: 5,18\% e 3,45\%, apenas.

\section{Considerações Finais}

Diante dos resultados apresentados, há uma percepção de que o conhecimento teórico sobre condução de tratamento nos internos de medicina está insuficiente, visto que as menores porcentagens foram observadas nas questões referentes a esse tema $\left(8^{\mathrm{a}}, 9^{\mathrm{a}}, 10^{\mathrm{a}}, 12^{\mathrm{a}}\right.$, principalmente). Visto que cada tipo de dislipidemia tem características diferentes, e há classes medicamentosas mais eficientes em tipos específicos, é importante que todos os médicos saibam classificar a dislipidemia para justamente alcançar a melhor condução e dar o melhor suporte possível ao paciente.

Ademais, observou-se que os entrevistados saberiam identificar um paciente com dislipidemia, pois nas questões referentes a solicitação, interpretação de exames laboratoriais e valores de referência para diagnóstica, os internos tiveram porcentagens positivas significativas $\left(3^{\mathrm{a}}, 4^{\mathrm{a}}, 6^{\mathrm{a}} \mathrm{e} 7^{\mathrm{a}}\right)$.

Foi visto, também, que há uma maior porcentagem de acerto entre os estudantes que já cursaram ou estão atualmente no ciclo de Atenção primária, quando comparados ao grupo que ainda está em curso. Assim, demonstra-se a importância da prática diária para o aprendizado do tema de dislipidemia, além da melhora no desempenho dos futuros médicos. De forma análoga, foi também visto um crescimento significativo na porcentagem de acertos quando comparado o $6^{\circ}$ ano do curso com o $5^{\circ}$ ano do curso de medicina. Tanto por mais alunos nesse primeiro grupo terem já cursado o ciclo de Atenção Básica, quanto 
por terem uma maior quantidade de experiência clínica na prática devido ao tempo maior no internato, demonstrando a importância de ciclos de internato de qualidade para a consolidação do conhecimento médico.

Conclui-se que, apesar de um conhecimento razoável na identificação de pacientes portadores de dislipidemia ou de seus fatores de risco, ainda há uma escassez de conhecimentos acerca da correta condução destes pacientes e das prerrogativas definidas nas diretrizes nacionais de dislipidemia. É mister que ocorra a buscar por tais conhecimentos visando sempre a melhor conduta possível para os pacientes, por todos os médicos, desde a atenção primária até a especializada. Considera-se que essa realidade está presente não apenas na faculdade de medicina estudada, mas em várias instituições pelo país. Essas podem contribuir para uma formação mais integral dos futuros médicos por meio de mudanças adaptativas curriculares, integrando a parte teórica dos estudos das diretrizes quando a parte prática. Novos estudos envolvendo tanto um número maior de estudantes, de múltiplas instituições, quanto da própria população médica também seriam interessantes visando uma análise mais aprofundada do conhecimento dos futuros profissionais e profissionais sobre o tema. Isso definiria com maior precisão as deficiências na formação médica e permitiria a formação de uma atenção mais especializada para sua correção.

\section{Referências}

Adamson, J., Beswick, A., \& Ebrahim, S. (2004). Is stroke the most common cause of disability? Journal Stroke Cerebrovascular Disease. $13(4): 171-177$.

Bertolami, M. C. (2017) Dislipidemias. Atualidades Médicas - Volume 1 - Edição nº 3

Bonfim, M. R.; et al. (2015). Tratamento das Dislipidemias com Estatinas e Exercícios Físicos: Evidências Recentes das Respostas Musculares. Arquivo Brasileiro Cardiologia. 104(4), 324-331.

Chou R. et al. (2018). Screening for Dyslipidemia in Younger Adults: A Systematic Review for the U.S. Preventive Services Task Force. Ann Intern Med. $165(8), 560-564$.

Gusso, G., Lopes, J. M. C., \& Dias, L. C. (2019); Tratado de Medicina da Família e Comunidade: princípios, formação e prática. $2^{\circ}$ Ed. Porto Alegre: Artmed Editora Ltda., 2019.

Hennekens, C. H. (2021); Overview of primary prevention of cardiovascular disease. https://www.uptodate.com/contents/overview-of-primary-prevention-ofcardiovasculardisease?search=Overview\%20of $\% 20$ primary $\% 20$ prevention $\% 20$ of $\% 20$ cardiovascular $\% 20$ disease, $\% 202020 \&$ source $=$ search_result $\&$ selectedTit le $=1 \sim 150 \&$ usage_type=default\&display_rank=1.

Faludi, A. A., Izar, M. C. O., \& SARAIVA, J. F. K.; (2017). Atualização da diretriz brasileira de dislipidemias e prevenção da Aterosclerose - 2017. Arquivos Brasileiros de Cardiologia, 109(2), 1-76.

Malachias, M.V.B., et al. (2016). $7^{\circ}$ Diretriz sobre Hipertensão Arterial da Sociedade Brasileira de Cardiologia. Sociedade Brasileira de Cardiologia, 107(3), 3

Malta, D. C., et al. (2016). Avanços do Plano de Ações Estratégicas para o Enfrentamento das doenças crônicas não transmissíveis no Brasil, $2011-2015$. Epidemiol. Serv. Saúde, 25(2), 373-390.

Pignone, M. (2021). Management of elevated low density lipoprotein-cholesterol (LDL-C) in primary prevention of cardiovascular disease. https://www.uptodate.com/contents/management-of-elevated-low-density-lipoprotein-cholesterol-ldl-c-in-primary-prevention-of-cardiovasculardisease?search=Dislipidemia\&topicRef=7573\&source=see_link.

Rosenson, R. S. (2020). Lab Interpretation: Abnormal lipid profile: High total cholesterol, low HDL cholesterol, high LDL cholesterol, or high triglycerides in adults. https://www.uptodate.com/contents/abnormal-lipid-profile-high-total-cholesterol-low-hdl-cholesterol-high-ldl-cholesterol-or-high-triglycerides-inadults?search=dislipidemia\&source=panel_search_result\&selectedTitle=5 150\&usage_type=panel\&display_rank=1 .

Santos, V., Dias, R. I., \& dos Santos, J. (2021). Conduta nutricional para prevenção e tratamento da aterosclerose. Revista Científica Do UBM, 23(45), 98-110.

Soares B.T., et al. (2018) Efeito do exercício físico sobre a dislipidemia. Rev. Aten. Saúde. 16(58), 12-16.

Souza, N. A. D., et al. (2019). Dislipidemia familiar e fatores associados a alterações no perfil lipídico em crianças. Ciência \& Saúde Coletiva [online]. 24(1), 323-332.

Suen, V. M. M., Durval R. F. \& De Almeida; C. A. (2017). Anais do XXI Congresso Brasileiro De Nutrologia. International Journal of Nutrology. 10(3), 3-14

Torres, N. C. P. Fatores nutricionais associados à hipertrigliceridemia em hipertensos do município de Maceió, Alagoas. Dissertação (Mestrado em Nutrição) - Faculdade de Nutrição, Programa de Pós-Graduação em Nutrição, Universidade Federal de Alagoas, Maceió, 2017.

Vijan, S. (2020). Screening for lipid disorders in adults. https://www.uptodate.com/contents/screening-for-lipid-disorders-inadults?topicRef=7573\&source=see_link.

Vos, T., et al. (2016). Global, regional, and national incidence, prevalence, and years lived with disability for 310 diseases and injuries, 1990-2015: a systematic analysis for the Global Burden of Disease Study 2015. Lancet. 388(10053), 1545-1602. 
Research, Society and Development, v. 11, n. 1, e20411124741, 2022

(CC BY 4.0) | ISSN 2525-3409 | DOI: http://dx.doi.org/10.33448/rsd-v11i1.24741

World Health Organization. (2017). Cardiovascular diseases. https://www.who.int/mediacentre/factsheets/fs317/en/.

Zhao, X. Q. (2020). Pathogenesis of atherosclerosis. https://www.uptodate.com/contents/pathogenesis-of-atherosclerosis?search= aterosclerosis\&source=search_result\&selectedTitle=1 150\&usage_type=default\&display_rank=1. 